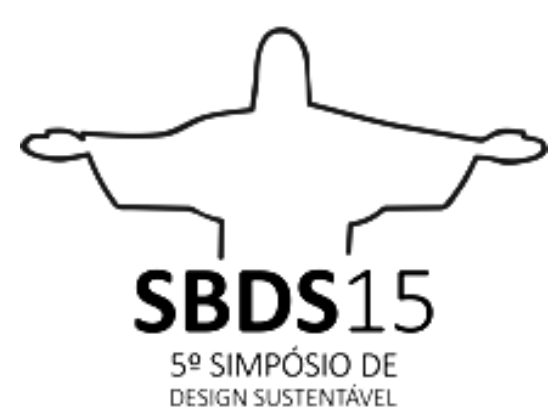

Rio de Janeiro, 11 de novembro a 13 de novembro de 2015

\title{
MATERIAL COMPÓSITO DE RESÍDUOS DE MDF E MDP ESTRUTURADOS EM MATRIZ DE PVC PARA PRODUTOS ALINHADOS PELO ECO-DESIGN
}

\author{
Fabiano André Trein \\ Universidade Federal da Paraná - Núcleo de Design e Sustentabilidade \\ fabianotrein@feevale.br \\ Aguinaldo dos Santos \\ Universidade Federal da Paraná - Núcleo de Design e Sustentabilidade \\ asantos@ufpr.br
}

\section{Resumo:}

Os problemas relacionados com as questões ambientais, como a poluição e geração de resíduos, e as suas consequências são, em muitos casos, ligada aos processos de produção. O consumo atual de madeira em grande escala confirmam as tendências para o uso de outro tipo de material, em substituição da madeira: resinas e aditivos especiais conhecidos como MDF (Medium Density Fiberboard). No entanto, devido à sua formulação química, o resíduo do processo de produção de MDF e serragem produzida pelo seu corte para a indústria moveleira é classificado como Classe I de resíduos - perigosos pela ABNT NBR 10004: 2004 e deve ter sua destinação obrigatória controlada. Neste sentido, a apresentação deste trabalho tem como objetivo apresentar a viabilidade técnica para a solução dos resíduos MDF através da produção de um novo material, utilizando os resíduos em uma matriz de PVC (cloreto de polivinila). Depois de um tratamento mecânico, a melhor fração obtida foi de $35 \%$ em peso de resíduos de MDF. Os principais resultados quantitativos obtidos são os seguintes: a densidade $1084 \mathrm{~kg} / \mathrm{m}^{3}$, a resistência à tracção superior a $40 \mathrm{MPa}$, o máximo esforço de flexão acima de $55 \mathrm{MPa}$, a resistência ao impacto de Charpy acima de 7,2 kJ / m2, absorção de água abaixo de 2,4\% e as características de não-inflamabilidade e de não propagação da chama. Sob o aspecto ambiental, a solução apresenta uma viabilidade técnica para a disposição dos resíduos obtendo produtos de ótima trabalhabilidade na indústria moveleira, automotiva e de agronegócios.

Palavras-chave: resíduo de MDF (Medium Density Fiberboard), Matriz de PVC, Sustentabilidade Ambiental, Eco-design. 


\begin{abstract}
The problems related to environmental issues such as pollution and waste generation, and its consequences are, in many cases, linked to production processes. The current consumption of wood on a large scale stablish the trends for the use of another kind of material, into substitution of the wood: resins and special additives known like MDF (Medium Density Fiberboard). However, due to its chemical formulation, the residue of the production process of MDF and sawdust produced by your cut for the furniture industry is classified as waste Class I - Hazardous by ABNT NBR 10004:2004 standard and must have its mandatory allocation controlled. In this sense, the presentation of this work aims to present the technical feasibility for the solution of MDF waste producing a new material, using the waste structured in a PVC matrix (polyvinyl chloride). After a mechanical processing, the fraction obtained the best characteristics was the one used $35 \%$ by weight of waste MDF. The main quantitative results obtained are: the bulk density $1084 \mathrm{~kg} / \mathrm{m}^{3}$, tensile strength above $40 \mathrm{MPa}$, the maximum bending stress above of $55 \mathrm{MPa}$, Charpy impact strength above 7.2 $\mathrm{kJ} / \mathrm{m}^{2}$, water absorption below $2.4 \%$ and the characteristics of nonflammability and non-flame propagation too. From an environmental aspect, the solution has a technical feasibility for the disposal of waste getting great workability products in the furniture, automotive and agribusiness industry.
\end{abstract}

KEYWORDS: MDF (Medium Density Fiberboard) waste, PVC matrix, Environmental sustainability, Eco-design.

\title{
1. INTRODUÇÃO
}

A preservação do meio ambiente é um assunto que se encontra no centro das discussões, nos contextos mundial, nacional e regional, em conferências e congressos, que tratam da sustentabilidade do Planeta e, consequentemente, da sustentabilidade humana na Terra, visando à manutenção qualitativa e quantitativa dos recursos naturais para o abastecimento de uma população em elevado crescimento.

Os problemas relacionados às questões ambientais como a poluição e a geração de resíduos, e suas consequências, estão, em muitos casos, associados aos processos produtivos. O melhor aproveitamento da matéria-prima ou a utilização dos resíduos da própria indústria como matéria-prima por meio de processos que incorporem o conceito de logística reversa e os princípios de gestão ambiental vem ganhando importância nas indústrias e instituições de pesquisas, pois, além dos benefícios ambientais, trazem vantagens econômicas às empresas (BLACKLEY, 1997).

Um indicador desta demanda ambiental é a adesão das empresas aos processos de certificações ambientais e florestais, solicitados pelos mercados, especialmente os internacionais, exigindo do setor produtivo a responsabilidade ambiental e social na exploração dos recursos florestais, com a máxima preservação possível destes recursos (CONROY, 2005). O atual consumo de madeira em grande escala, pelos diversos setores da sociedade, faz com que surjam discussões e questionamentos sobre os impactos dos resíduos madeireiros ao ecossistema, instigando a ciência florestal no desenvolvimento de pesquisas sobre soluções 
mitigadoras dos impactos ambientais gerados nos processos produtivos, onde se tem a matéria-prima madeira ou painéis compensados de madeira como principais componentes do processo.

A necessidade de máximo aproveitamento dos resíduos das indústrias que utilizam produtos do setor florestal, visando à otimização do uso da matéria-prima, requer soluções com foco na busca pelo melhor aproveitamento desse tipo de material, viabilizando o consumo dos produtos gerados (LEITE, 2009).

Neste contexto, existe a demanda por soluções que viabilizem a utilização de resíduos de painéis de madeira, oriundos do setor moveleiro, da construção civil e outros setores onde ocorre elevado descarte desses produtos, para compor novos materiais que fechem o ciclo produtivo e possam ser reaproveitados. Essas soluções podem favorecer o melhor aproveitamento da matéria-prima, proporcionado maior valor agregado ao produto bem como novas propriedades que melhoram 0 desempenho dos mesmos (ANDRADY, 2003). Soluções que visem avaliar os resíduos provenientes de painéis compensados, MDF e aglomerados, estes ultimamente conhecidos como MDP, utilizados na fabricação de móveis, na construção civil e em outros segmentos, incorporando-os na produção de novos painéis de madeira aglomerada, são importantes, pois atendem ao compromisso ambiental e aos interesses econômicos da indústria.

A importância desta pesquisa e aplicação prática está na técnica produtiva e na análise qualitativa dos produtos originados de resíduos de painéis, incentivando a produção sustentável, atendendo a logística reversa e a viabilidade econômica, favorecendo, desta forma, o meio ambiente e a sociedade. O presente estudo trata dos resíduos de painéis de MDF e MDP produzidos por essa e outras descritas no estado da técnica para o reaproveitamento dentro da própria indústria moveleira que a gerou. A presente invenção não se utiliza de energia de aglutinação por micro-ondas estimulando um processo químico.

Neste sentido, a apresentação desta pesquisa tem por objetivo apresentar a viabilidade técnica para a solução dos resíduos de MDF/MDP gerados na indústria moveleira através da produção de um novo material, que na forma de perfis, com diversas formas, podem ser utilizados na mesma indústria na forma de decks, pisos, roda-pés, tubos e peças técnicas.

É fruto da presente pesquisa o processo de produção e produto constituído por material compósito de resíduos de MDF e MDP estruturados em matriz de PVC (policloreto de vinila) com desempenho para uso específico na indústria moveleira e da construção civil. Os resíduos dos painéis de MDF e MDP, vindos dos processos de corte, furação e lixamento da indústria de móveis, principalmente, podem ser facilmente reaproveitados pelo presente produto.

\section{DESENVOLVIMENTO}

\subsection{Concepção da Produção de Móveis e Geração de Resíduos}

Atualmente, uma tendência para produção de móveis é a utilização de painéis de madeira compensada, ou seja, formada de cavacos, chips e fibras de madeira, prensada a altas temperaturas com resinas sintéticas, usualmente fenólicas, uréicas e aditivos especiais, produzindo produtos conhecidos por MDF (Medium Density Fiberboard ou painel de fibras de média densidade) e MDP (Medium Density 
Particleboard ou painel de partículas de média densidade). Facilitando as operações produtivas na indústria de móveis pela padronização do produto. A principal diferença entre os dois é que no painel de MDP são utilizadas partículas de madeira em camadas, ficando as mais finas na superfície e as mais delgadas no miolo. Já no MDF, aglutinam-se fibras de madeira. Porém, ambos são classificados como Painéis de Madeira de Média Densidade.

Os painéis de madeira surgiram da necessidade de amenizar a anisotropia e a instabilidade dimensional da madeira maciça, diminuir seu custo e melhorar as propriedades isolantes, térmicas e acústicas. Adicionalmente, suprem uma necessidade reconhecida no uso da madeira serrada e ampliam a sua superfície útil, através da expansão de uma de suas dimensões (a largura), para, assim, otimizar a sua aplicação. Os painéis de madeira podem ser definidos como produtos compostos de elementos de madeira como lâminas, sarrafos, partículas e fibras, obtidos a partir da redução da madeira sólida e reconstituídos através de ligação adesiva.

As utilizações dos painéis de madeira estão diretamente associadas às propriedades físicas e mecânicas dos mesmos. As restrições técnicas para o uso e a aplicação de diferentes tipos de painéis de madeira envolvem características como resistência, uso interior ou exterior, uniformidade da superfície, tolerância à usinagem, resistência à fixação de parafusos, entre outros. Diferentes tipos de painéis de madeira podem sobrepor tais restrições técnicas. Os principais usos e aplicações dos painéis de madeira estão associados principalmente aos segmentos da construção civil e de móveis. Os painéis de madeira podem ser diferenciados por suas propriedades, as quais permitem aplicações nesses dois segmentos.

Os resíduos desses painéis de madeira, especificamente MDF e MDP, possuem potencial econômico viável, ainda pouco explorado pelas empresas. A alternativa de seu aproveitamento na produção de novos painéis é uma ideia que vem ao encontro do apelo social atual, favorável à adoção de materiais que provoquem o mínimo impacto ambiental ao Planeta, conforme figura 1.

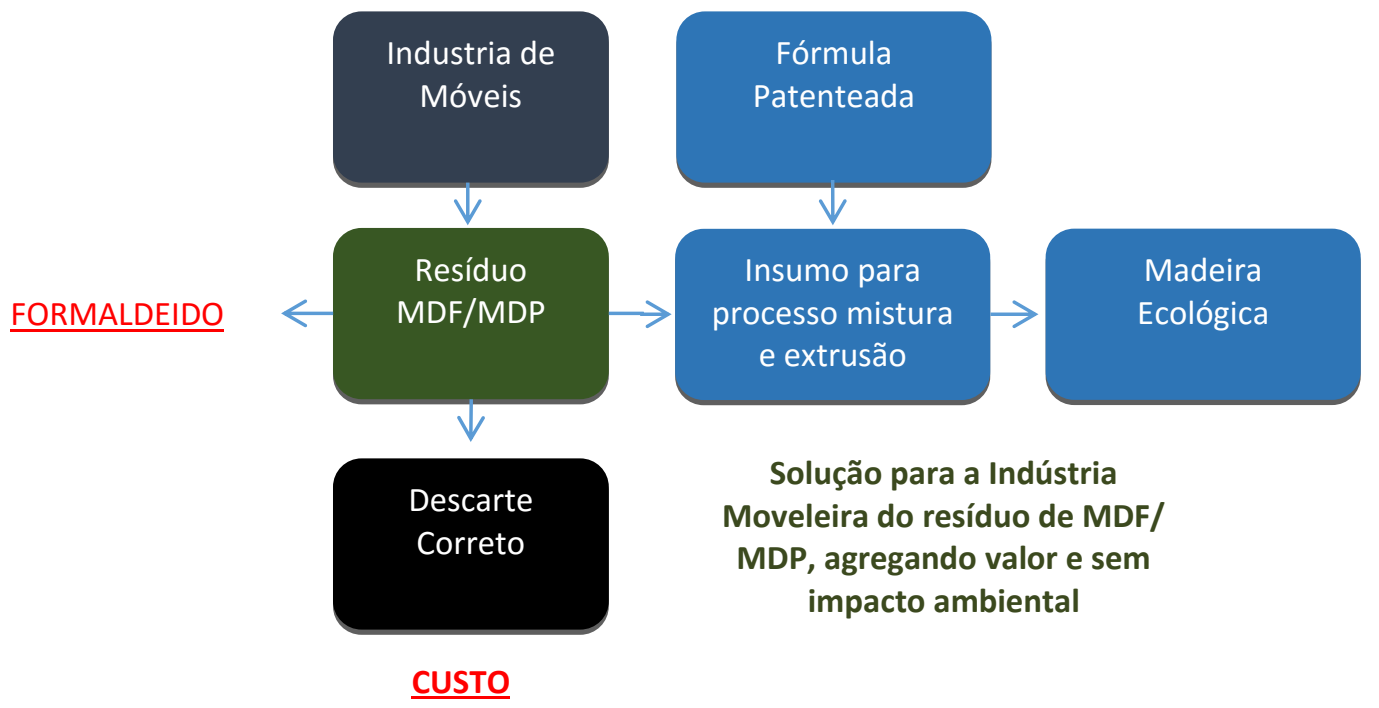

Figura 1 - Logística reversa do Pó de MDF.

Fonte: Elaborado pelo autor, com base na pesquisa realizada. 
Muitas empresas que utilizam painéis de madeira como matéria-prima nos processos produtivos, principalmente as indústrias moveleiras e a de construção civil, destinam os resíduos desses painéis (aparas, retalhos ou painéis danificados) ao ecossistema, em aterros industriais, ou à queima para geração energética, podendo causar impactos ambientais ao solo, aos recursos hídricos, à fauna, à flora e a atmosfera devido à composição química encontrada nos produtos, como resinas, parafina, extrativos da própria madeira, entre outras substâncias. Este material, ainda visto como resíduo, apresenta grande potencial como matéria-prima para produção de novo painéis. O destino inadequado, a deposição indevida dos resíduos no meio ambiente ou mesmo a deposição em aterros industriais provocam, além de danos ambientais, prejuízo econômico, onde a logística reversa, o reaproveitamento ou o coprocessamento adequados desses resíduos poderiam tornar-se lucrativo à empresa geradora. A necessidade de máximo aproveitamento dos resíduos das indústrias que utilizam produtos do setor florestal, visando à otimização do uso da matéria-prima, requer soluções com foco na busca pelo melhor aproveitamento desse tipo de material, viabilizando o consumo dos produtos gerados (AL SALEM et. al., 2010).

Os resíduos sólidos gerados normalmente na forma de pó pela cadeia produtiva moveleira são provenientes, em sua maioria, das operações de usinagem dos painéis, principalmente, corte, furação e lixamento. Dessa forma, em diversas etapas do processamento dos painéis, desde a fabricação até a operação de lixamento de qualquer peça de um móvel, são gerados resíduos em diferentes proporções e com diferentes características.

De acordo com Silva e Figueiredo (2010), para criar um produto com critérios ecológicos ou fazer um redesign de um produto já existente, o designer deve estar atento às decisões que precisa tomar em todas as fases do ciclo de vida do produto: pré-produção, produção, uso, descarte, reciclagem, reuso, etc., para que a minimização do impacto ambiental do produto ocorra de forma a atender seus objetivos. Com isso, é possível perceber que os principais objetivos de produtos desenvolvidos com foco no ecodesign são o econômico e o ambiental, conforme pode ser visto na figura 2.

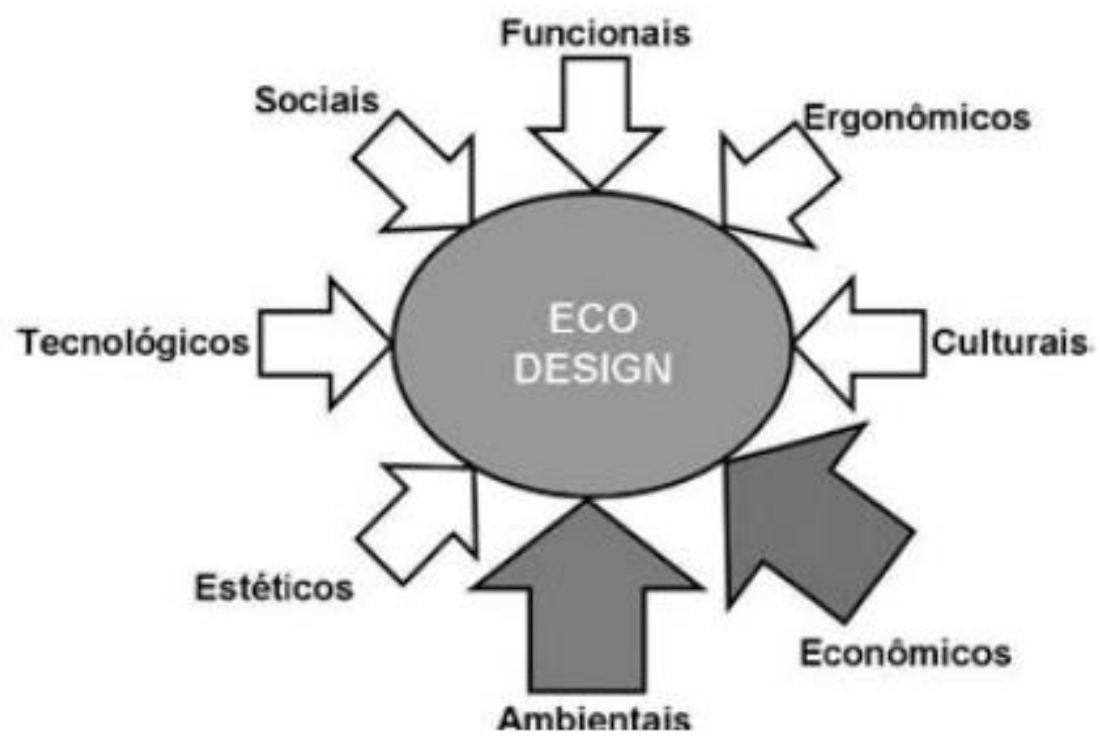

Figura 2 - Aspectos do Eco-Design.

Fonte: Pazmino, 2007. 
O potencial de reaproveitamento desses resíduos pode ser estimado pela produção crescente de painéis e segundo o estudo setorial da Associação Brasileira da Indústria de Madeira Processada Mecanicamente - $A B I M C l$ (2009), a indústria brasileira de compensados produziu aproximadamente 2,5 milhões de metros cúbicos de compensados de coníferas e de folhosas em 2008. Somem-se a isso as informações da Associação Brasileira da Indústria de Painéis de Madeira - ABIPA (2010), as empresas fabricantes de MDF têm capacidade nominal instalada de 4,1 milhões de metros cúbicos ao ano, enquanto as empresas fabricantes de painéis MDP têm capacidade nominal instalada de 4,8 milhões de metros cúbicos ao ano. Revelando que o problema dos resíduos nessa indústria é crescente e a solução de reaproveitamento dos resíduos de MDF e MDP cada vez mais urgente (ABIPLAST, 2010).

\subsection{Metodologia e Desenvolvimento da Inovação}

O processo para a produção de perfis de material compósito de resíduos de MDF e MDP, na forma de pó oriundo do processo produtivo seguiu as etapas conforme Fluxograma de Processo constante na figura 3.

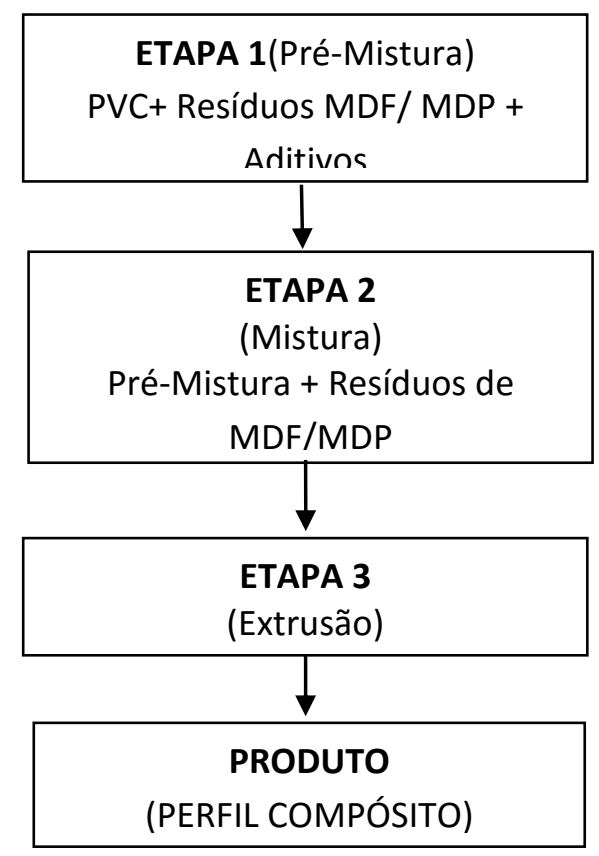

Figura 3 - Fluxograma de Processo.

Fonte: Elaborado pelo autor, com base na pesquisa realizada.

Iniciou-se a Etapa 1 com a preparação da formulação com PVC, conforme descrito na tabela 1 , utilizada para perfis rígidos extrudados ou injetados. Utilizou-se uma mistura, ou blend, de resinas de PVC com diferentes pesos moleculares, utilizando-se também resíduos de PVC, possibilitando a produção de perfis com diferentes índices de dureza para diferentes usos na indústria moveleira ou da construção civil. Os resíduos de PVC foram oriundos de diferentes indústrias, principalmente àquelas que manipulam e produzem produtos de PVC como esquadrias, perfis e portas (LA MANTIA, 2002). 
Adicionou-se a essas resinas de PVC, inclusive aos resíduos, ainda: estabilizantes térmicos, lubrificantes térmicos, auxiliar de processamento de extrusão e plastificantes. Os componentes da formulação foram adicionados em uma câmara cilíndrica de um misturador intensivo de PVC com resfriador vertical, ou outro que possa misturar a formulação sem plastificá-la, através de resfriamento da massa.

Tabela 1: Formulação de PVC (Pré-Mistura) - Etapa 1.

\begin{tabular}{c|c|c}
\hline Componentes & $\begin{array}{c}\text { Faixa de Utilização } \\
\text { \% em peso }\end{array}$ & $\begin{array}{c}\text { Melhor Utilização } \\
\text { \% em peso }\end{array}$ \\
\hline Resina de PVC para injeção & 47,7 a 97,7 & 75 \\
\hline $\begin{array}{c}\text { Resina de PVC de baixo peso } \\
\text { molecular ou Resíduos de PVC }\end{array}$ & 0,5 a 37,7 & 18 \\
\hline Estabilizante Térmico & 0,7 a 6,0 & 5 \\
\hline Lubrificante Externo & 0,3 & 0,3 \\
\hline Auxiliar de processamento & 0,1 a 1,0 & 1 \\
\hline Plastificante & 0,1 a 15,0 & 0,7 \\
\hline
\end{tabular}

Fonte: Elaborado pelo autor, com base na pesquisa realizada.

Após essa pré-mistura de preparação da resina, foi executada a Etapa $2 \mathrm{com}$ a mistura com os resíduos de MDF/MDP na forma de pó. A pré-mistura foi misturada no mesmo misturador intensivo com os resíduos de MDF/MDP na forma de pó nas proporções estabelecidas na tabela 2 a seguir.

Tabela 2: Formulação da Pré-Mistura com Resíduos de MDF/MDP - Etapa 2.

\begin{tabular}{c|c|c}
\hline Componente & $\begin{array}{c}\text { Faixa de Utilização } \\
\text { \% em peso }\end{array}$ & $\begin{array}{c}\text { Melhor Utilização } \\
\text { \% em peso }\end{array}$ \\
\hline Pré-Mistura (Tabela 1) & 40 a 85 & 65 \\
\hline $\begin{array}{c}\text { Resíduos de MDF/MDP } \\
\text { (pó) }\end{array}$ & 1560 & 35 \\
\hline
\end{tabular}

Fonte: Elaborado pelo autor, com base na pesquisa realizada.

Para a preparação das composições foi utilizado o resfriador horizontal onde foram misturados mecanicamente por cerca de 20 segundos em baixa rotação o pó de resíduos de MDF/MDP (Etapa 2) com a formulação da pré-mistura de PVC rígido (Etapa 1) nas devidas proporções, de modo a fixar uma fração mássica de resíduos de MDF/MDP na composição.

Após mistura e resfriamento, os compostos dry-blend foram granulados em uma extrusora mono ou dupla rosca (relação comprimento/ diâmetro - L/D 25).

Portanto, foi importante existir um balanço entre o benefício proveniente dos resíduos de MDF/MDP e o prejuízo causado pelo plastificante nas propriedades mecânicas para se obtiver um material de desempenho adequado à indústria moveleira e da construção civil. A fração que obteve as melhores características foi a de $35 \%$ em massa de resíduos de MDF/MDP, mas descobriu-se que outras faixas de utilização também possuem características de desempenho para uso, pelo menos, na indústria de móveis e da construção civil.

Após a mistura da Etapa 2, executou-se a Etapa 3, onde o material granulado seguiu para extrusão do perfil em uma mono ou dupla rosca (relação comprimento/ 
diâmetro - L/D 25) com passo de rosca adequado para uso de PVC, para produção do perfil de material compósito em diversos formatos para uso como, por exemplo, rodapé, substituto de madeira para bancos e diversos outros desenhos e formas. 0 material também pode ser injetado para a produção de peças de tamanho e formas diferenciadas.

As principais aplicações visualizadas inicialmente são apresentadas nas figuras $4,5,6$ e 7 .

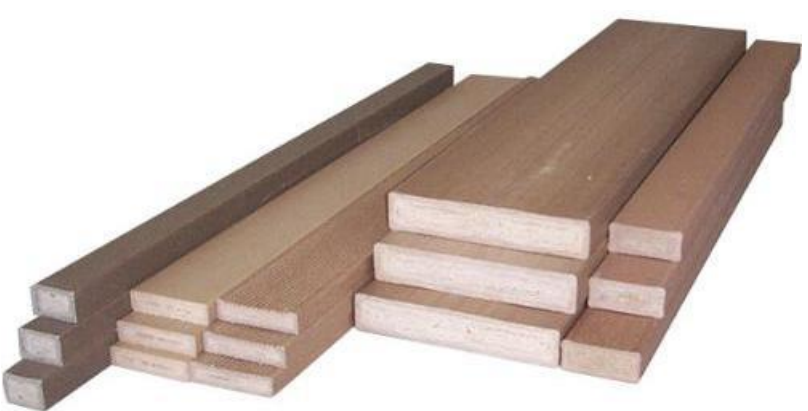

Figura 4 - Chapas estrusadas em diversos tamanhos. Fonte: Elaborado pelo autor, com base na pesquisa realizada.

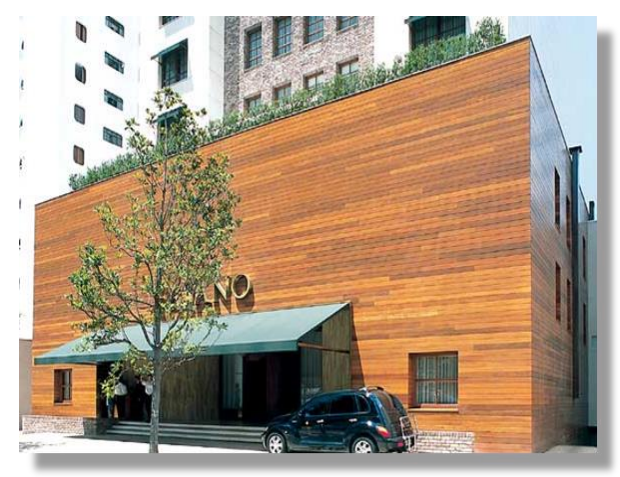

Figura 6 - Fachadas.

Fonte: Elaborado pelo autor, com base na pesquisa realizada.

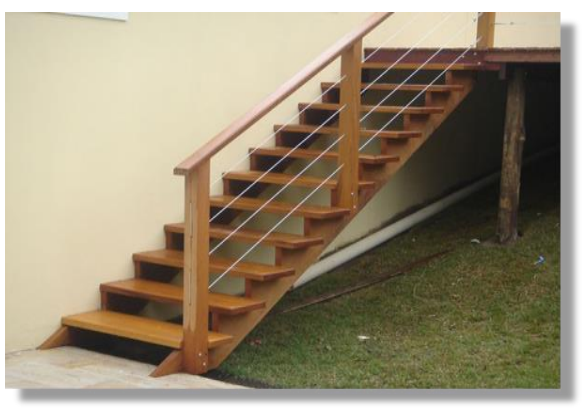

Figura 5 - Escadas externas.

Fonte: Elaborado pelo autor, com base na pesquisa realizada.

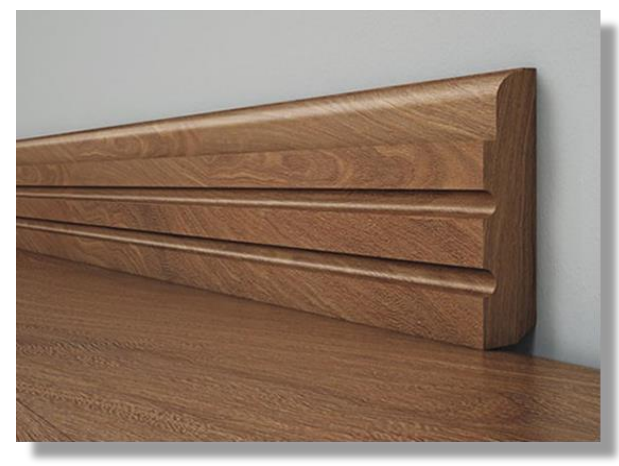

Figura 7 - Roda-pé.

Fonte: Elaborado pelo autor, com base na pesquisa realizada.

\subsection{Principais Resultados Qualitativos}

De uma forma geral, as peças produzidas apresentaram as seguintes características em relação aos seus concorrentes atuais:

- Maior resistência às ações da natureza: sol, frio, chuva, calor, cupins;

- Dispensa-se o uso de pesticidas;

- Menos peso por $\mathrm{m}^{2}$ da área de cobertura em relação às réguas de madeira da concorrência;

- Possibilidade de personalização das réguas (tamanhos, formas e cores);

- Facilidade de embalagem e transporte;

- Não há necessidade de ser pintada, podendo ser pigmentada;

- Não forma farpas, não racha, nem empena pelo ressecamento ou envelhecimento; 
- Madeira convencional precisa de manutenção em 2 anos, o que acarreta custo adicional enquanto nosso produto tem vida útil de mais de 50 anos.

Os produtos obtidos após o processo de extrusão ou injeção podem ser visualizados nas figuras $8,9,10,11,12$ e 13 .

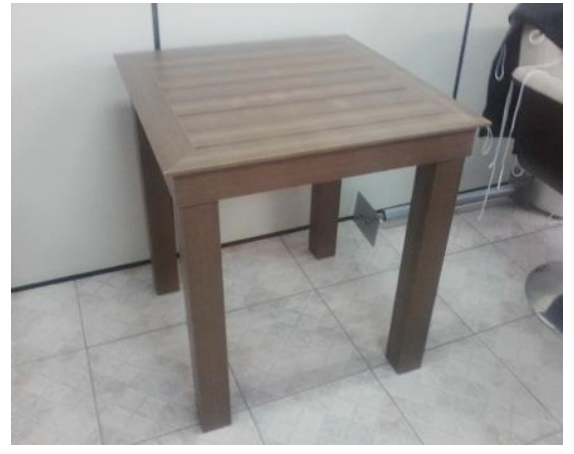

Figura 8 - Móveis para áreas externas.

Fonte: Elaborado pelo autor, com base na pesquisa realizada.

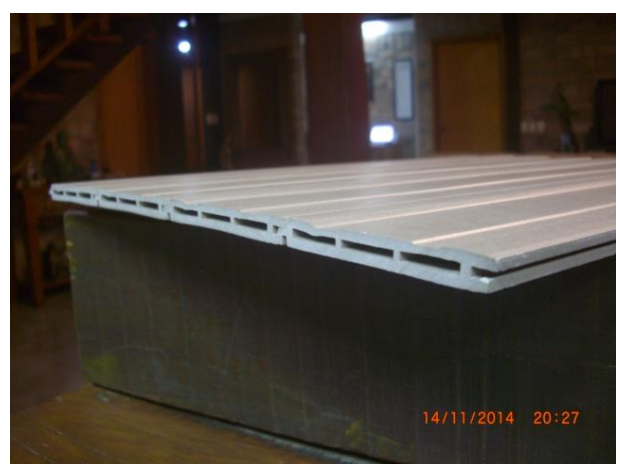

Figura 10 - Decks e pisos.

Fonte: Elaborado pelo autor, com base na pesquisa realizada.

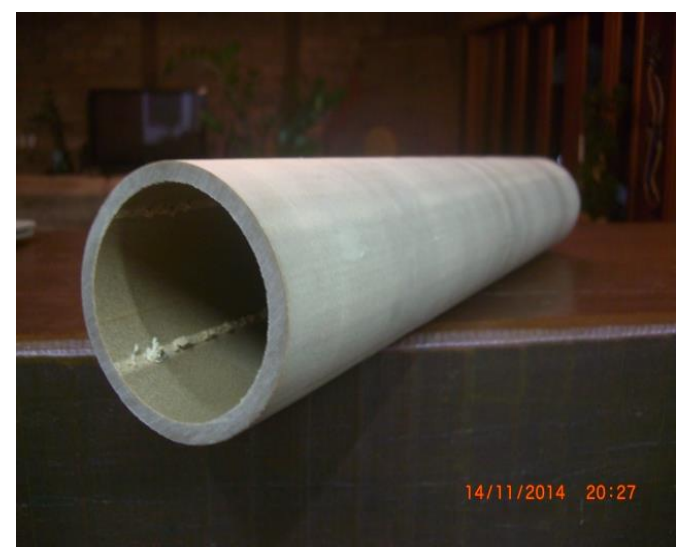

Figura 12 - Tubos e estruturas.

Fonte: Elaborado pelo autor, com base na pesquisa realizada.

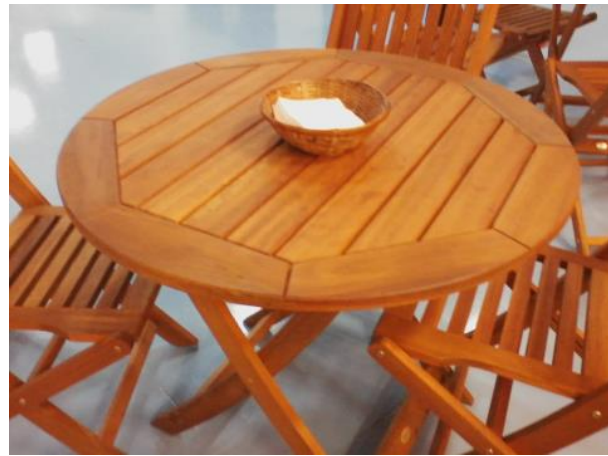

Figura 9 - Móveis para áreas internas.

Fonte: Elaborado pelo autor, com base na pesquisa realizada.

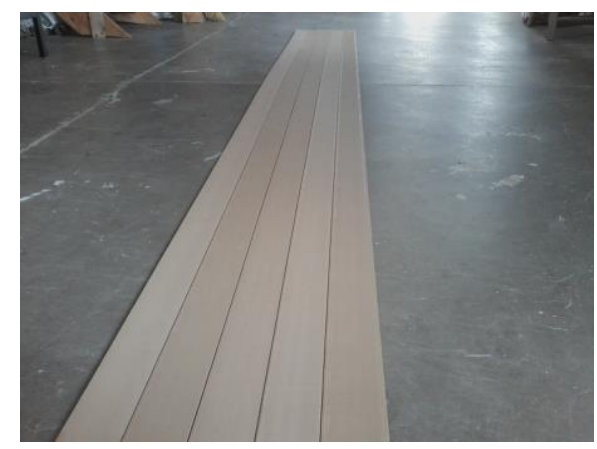

Figura 11 - Decks e pisos.

Fonte: Elaborado pelo autor, com base na pesquisa realizada.

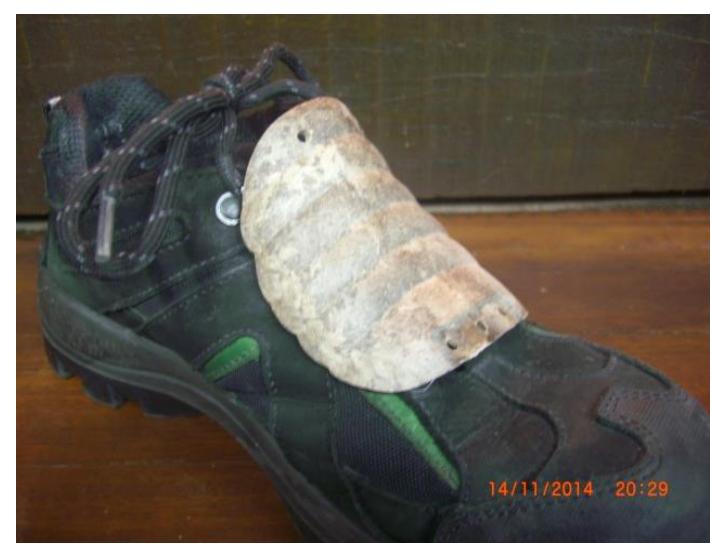

Figura 13 - Peças técnicas.

Fonte: Elaborado pelo autor, com base na pesquisa realizada. 


\subsection{Principais Resultados Quantitativos}

0 produto obtido a partir desse processo de 3 etapas obteve o seguinte desempenho físico-mecânico, conforme descrito na tabela 3 a seguir.

Tabela 3: Ensaios de Desempenho realizados no SENAI - CETEPO - São Leopoldo - RS realizados em 22/5/2013 Relatório 559/2013.

\begin{tabular}{c|c|c}
\hline Ensaio & Norma & Valores Característicos \\
\hline $\begin{array}{c}\text { Resistência à Tração em } \\
\text { Materiais Plásticos - } \\
\text { Tensão na Ruptura }\end{array}$ & ASTM D638 & 17 a $41 \mathrm{MPa}$ \\
\hline $\begin{array}{c}\text { Tensão na Máxima } \\
\text { Flexão }\end{array}$ & ASTM D790 - Método A & 24 a $58 \mathrm{MPa}$ \\
\hline $\begin{array}{c}\text { Densidade relativa } \\
\text { Resistência ao Impacto } \\
\text { Charpy }\end{array}$ & ASTM D792 - Método A & 1.030 a $1.138 \mathrm{~kg} / \mathrm{m}^{3}$ \\
\hline $\begin{array}{c}\text { Inflamabilidade em } \\
\text { Materiais Plásticos }\end{array}$ & ISO 709 & 4,8 a $7,2 \mathrm{~kJ} / \mathrm{m}^{2}$ \\
\hline $\begin{array}{c}\text { Absorção de água em } \\
\text { materiais plásticos a } \\
23^{\circ} \mathrm{C}\end{array}$ & ASTM D570 & Não Inflamável \\
\hline
\end{tabular}

Fonte: Elaborado pelo autor, com base na pesquisa realizada.

Verificou-se que perfis de materiais compósitos para uso na indústria moveleira devem possuir maior massa específica e maior resistência mecânica, sem prejudicar sua estabilidade dimensional. Os materiais compósitos obtidos são facilmente usinados a fim de possibilitarem a introdução e aprisionamento de parafusos e fornecer após processamento um ótimo acabamento. As características descobertas com esse novo material compósito dão ao mesmo, ótimas características de desempenho.

Destaca-se igualmente a baixa absorção de água, média de 2,40\%, característica importante para uso externo dos materiais compósitos, e a não inflamabilidade e a não propagação de chamas do mesmo. Essas características fornecem ao produto compósito um desempenho excepcional para uso na indústria de móveis e da construção civil.

\section{CONCLUSÃO}

As oportunidades apresentadas e as peças produzidas a partir do material compósito conforme o processo descrito e extrusado ou injetado na forma de um perfil, com diversas formas, apresentam desempenho e características adequadas para o uso na indústria da construção civil e moveleiro. 
Destacam-se as excelentes características físico-mecânicas, tais como a resistência à tração, resistência à água, resistência à flexão, não inflamabilidade, não propagação de chamas, ótima dispersão e homogeinização do pó de MDF/MDP na matriz de PVC e densidade relativa.

Elucidam-se ainda as características de manuseio e trabalhabilidade das peças obtidas, tais como: ótima fixação e penetração de parafusos e pregos, corte, usinagem e furação.

Adicionalmente, sob o aspecto ambiental e sustentável destaca-se o reprocessamento do rejeito, que antes era descartado no meio ambiente, pode ser reciclado em um novo material compósito, minimizando os efeitos ao meio ambiente e o impacto antrópico.

O produto já está em fase de produção de lotes piloto nos segmentos da construção civil, automotivo, mobiliário e agronegócio, tendo seus direitos depositados em nome do autor em patente junto ao Instituto Nacional da Propriedade Intelectual - INPI através dos registros BR 1020130143863 e BR 1320130175713.

Como trabalhos futuros, destaca-se a aplicação deste novo material no desenvolvimento de novos perfis alinhados ao Eco-design e à substituição dos materiais tradicionais, como o plástico e a madeira.

\section{REFERÊNCIAS}

AI SALEM, S.M. et. al. Recycling and recovery routes of plastic solid waste (PSW): a review. Waste Management, v. 29. 2009, p. 2625 - 2643.

ANDRADY, A.L. Plastics and the environment. New Jersey: John Wiley \& Sons, Inc., 2003.

Associação Brasileira da Indústria de Madeira Processada Mecanicamente - ABIMCI. Estudo Setorial. (2009).

Associação Brasileira da Indústria de Painéis de Madeira - ABIPA. Estudo Anual Setorial. (2010).

Associação Brasileira da Indústria do Plástico - ABIPLAST. Perfil 2013 - Indústria Brasileira de Transformação de material plástico. Disponível em http://file.abiplast.org.br/download/links links\%202014/perfil2013 abiplast final web.pdf. Acesso em: 11 outubro 2014.

Associação Brasileira de Normas Técnicas. NBR 10004: resíduos sólidos - classificação. Rio de Janeiro, 2004.

BLACKLEY, D.C. Polymer lattices. Science and Technology, 2nd Edition, 1997.

CONROY, A. et. al. Composite recycling in the construction industry. Composites: Part A v. 37. $1216-1222.2005$. 
INSTITUTO NACIONAL DE METROLOGIA - INMETRO, Manual para implementação do REACH - Barreiras Técnicas às Exportações. 1a Edição. Rio de Janeiro. 2009.

LA MANTIA, F. Handbook of Plastics Recycling. Shropshire, UK: Rapra Technology Limited. 2002.

LEITE, P. R. Logística Reversa: meio ambiente e competitividade, São Paulo, Pearson Prentice Hall, 2009.

LIDDLE, B. T. Construction for Sustainability and the Sustainability of the Construction Industry. In: CIB TG 16 Sustainable Construction. Proceedings. Tampa, Florida, p.47-56, 1994.

PAZMINO, A. V. Uma reflexão sobre Design Social, Eco-Design e Design Sustentável. I Simpósio Brasileiro de Design Sustentável. Anais. Curitiba, 2007.

RIBEIRO, D. V.; MORELLI, M.R. Resíduos sólidos: problema ou oportunidade? Rio de Janeiro: Interciência, 2009.

SILVA,R. C. et. al. Relatório sobre a Geração de Resíduos Sólidos Industriais no Estado do Rio Grande do Sul: FEPAM e FNMA, 27p. 2006. 